\title{
Triangle anomalies and nonrelativistic Nambu-Goldstone modes of generalized global symmetries
}

\author{
Noriyuki Sogabe and Naoki Yamamoto \\ Department of Physics, Keio University, Yokohama 223-8522, Japan
}

(Received 20 March 2019; published 6 June 2019)

\begin{abstract}
In massless QCD coupled to QED in an external magnetic field, a photon with the linear polarization in the direction of the external magnetic field mixes with the charge neutral pion through the triangle anomaly, leading to one gapless mode with the quadratic dispersion relation $\omega \sim k^{2}$ and one gapped mode. We show that this gapless mode can be interpreted as the so-called type-B Nambu-Goldstone (NG) mode associated with the spontaneous breaking of generalized global symmetries and that its presence is solely dictated by the anomalous commutator in the symmetry algebra. We also argue a possible realization of such nonrelativistic NG modes in 3-dimensional Dirac semimetals.
\end{abstract}

DOI: 10.1103/PhysRevD.99.125003

\section{INTRODUCTION}

Spontaneous symmetry breaking (SSB) is one of the most important notions in modern physics that explains various physical phenomena from superfluidity to the origin of hadron masses. In general, when a continuous global symmetry is spontaneously broken, a gapless collective excitation called the Nambu-Goldstone (NG) mode emerges [1-3]. In a relativistic system with Lorentz invariance, the NG mode has the dispersion relation $\omega=k$ and the number of NG modes, $N_{\mathrm{NG}}$, is equal to the number of broken symmetry generators, $N_{\text {BS }}$ [4]. This is not always the case in a system without Lorentz invariance, where the NG mode can have the dispersion $\omega \sim k^{2}$ and $N_{\mathrm{NG}}$ can be smaller than $N_{\mathrm{BS}}$ [5-7].

Whether an NG mode has the linear or quadratic dispersion relation is classified by the quantity $\rho_{a b} \equiv\left\langle\left[Q_{a}, Q_{b}\right]\right\rangle \neq 0$, with $Q_{a}$ broken symmetry generators and the expectation value taken in the vacuum: the NG mode characterized by nonvanishing $\rho_{a b}$ typically has the quadratic dispersion relation and is called type- $\mathrm{B}$, while the rest typically has the linear dispersion relation and is called typeA. The generic counting rule for the numbers of the type-A and type-B NG modes, $N_{\mathrm{A}}$ and $N_{\mathrm{B}}$, is summarized as [8-10]

$$
\begin{aligned}
N_{\mathrm{NG}} & =N_{\mathrm{A}}+N_{\mathrm{B}}, \\
N_{\mathrm{A}} & =N_{\mathrm{BS}}-2 N_{\mathrm{B}}, \\
N_{\mathrm{B}} & =\frac{1}{2} \operatorname{rank} \rho_{a b} .
\end{aligned}
$$

Published by the American Physical Society under the terms of the Creative Commons Attribution 4.0 International license. Further distribution of this work must maintain attribution to the author(s) and the published article's title, journal citation, and DOI. Funded by SCOAP .
So far, the notions of the SSB and NG modes have been mostly applied to the ordinary symmetries for pointlike objects. Yet, these notions can be generalized to symmetries for extended objects (such as strings and branes), called the generalized global symmetries or higher-form symmetries [11]. In particular, the massless photon can be understood as a type-A NG mode associated with the SSB of a generalized global symmetry. More recently, it has been shown that photons with the quadratic dispersion relation (or nonrelativistic photons) appear in the axion electrodynamics with a spatially varying and periodic $\theta$ term and that it may be interpreted as a type-B NG mode $[12,13]$. Such a situation is indeed expected to be realized in dense nuclear or quark matter in a magnetic field $[12,14]$ and a periodic array of topological and normal insulators [15] (see also Ref. [16]).

In this paper, we show that the emergence of the type-B NG mode of generalized global symmetries is more generic than previously thought and it appears in a much more simple setup: strongly interacting massless Dirac fermions coupled to a dynamical U(1) gauge field in a background magnetic field. One example is QCD coupled to QED in the external magnetic field. In particular, translational symmetry breaking by the spatially varying $\theta$ term in the previous examples $[12,14,15]$, which was conjectured in Ref. [14] to be the only way to realize the nonrelativistic photon, is not necessary in this case.

Our main purpose of this paper is to show further that the presence of this type-B NG mode is solely dictated by the anomalous commutator related to the triangle anomaly in the symmetry algebra. In this sense, this phenomenon is universal depending on the SSB of generalized global symmetries, SSB of chiral symmetry, and the presence of the triangle anomaly. As we shall also discuss, this type-B NG mode should be experimentally testable, e.g., in Dirac semimetals in 3-dimensional solids. 


\section{EXAMPLE}

\section{A. Setup}

As an example, we consider massless two-flavor QCD coupled to QED in an external homogeneous magnetic field $\boldsymbol{B}_{\text {ex }}$. A similar setup with finite quark mass was previously studied in Ref. [17]. The presence of the external magnetic field explicitly breaks chiral symmetry down to $\mathrm{U}(1)_{\mathrm{V}}^{\tau_{3}} \times \mathrm{U}(1)_{\mathrm{A}}^{\tau_{3}}$, which is the invariance under the transformation $q \rightarrow \mathrm{e}^{\mathrm{i} \alpha_{V} \tau_{3}} \mathrm{e}^{\mathrm{i} \alpha_{\mathrm{A}} \tau_{3} \gamma_{5}} q$, with $\tau^{3}$ being one of the SU(2) generators. This partial chiral symmetry is spontaneously broken to $\mathrm{U}(1)_{\mathrm{V}}^{\tau_{3}}$ in the vacuum. The low-energy degrees of freedom below the energy scale of charged pions are the charge neutral pion $\pi^{0}$ and massless photons, which we shall focus on below.

At the leading order in derivatives, the effective Lagrangian for $\pi^{0}$ and electromagnetic field $F^{\mu \nu}$ is

$$
\mathcal{L}=\frac{1}{2} \partial_{\mu} \pi^{0} \partial^{\mu} \pi^{0}-\frac{1}{4} F_{\mu \nu} F^{\mu \nu}-\frac{C}{4} \frac{\pi^{0}}{f_{\pi}} F_{\mu \nu} \tilde{F}^{\mu \nu},
$$

where the last term is the Wess-Zumino-Witten (WZW) term $[18,19]$ that accounts for the triangle anomaly in this low-energy effective theory with $C=1 /\left(4 \pi^{2}\right), f_{\pi}$ the pion decay constant, and $\tilde{F}^{\mu \nu}=\frac{1}{2} \epsilon^{\mu \nu \alpha \beta} F_{\alpha \beta}$. (The factor $1 / 4$ in front of the WZW is just for later convenience.) We assume the local charge neutrality in the vacuum.

Let us consider the fluctuations of $E^{i}=-F^{0 i}$, $B^{i}=-\tilde{F}^{0 i}$, and $\pi^{0}$ around the QCD vacuum in the background magnetic field. We set $\boldsymbol{B}=\boldsymbol{B}_{\mathrm{ex}}+\delta \boldsymbol{B}, \boldsymbol{E}=\delta \boldsymbol{E}$, and $\pi^{0}=\delta \pi^{0}$ and we focus on the first order in fluctuations. The equations of motion for $\pi^{0}$ and electromagnetic fields are

$$
\begin{gathered}
\left(\partial_{t}^{2}-\nabla^{2}\right) \delta \pi^{0}=\frac{C}{f_{\pi}} \boldsymbol{B}_{\mathrm{ex}} \cdot \delta \boldsymbol{E}, \\
\boldsymbol{\nabla} \cdot \delta \boldsymbol{E}=-\frac{C}{f_{\pi}} \boldsymbol{\nabla} \delta \pi^{0} \cdot \boldsymbol{B}_{\mathrm{ex}}, \\
-\partial_{t} \delta \boldsymbol{E}+\boldsymbol{\nabla} \times \delta \boldsymbol{B}=\frac{C}{f_{\pi}} \partial_{t} \delta \pi^{0} \boldsymbol{B}_{\mathrm{ex}},
\end{gathered}
$$

respectively. The right-hand sides of Eqs. (6) and (7) are the anomalous charge and current induced by the pion fluctuation. In particular, the latter is the counterpart of the chiral magnetic effect (CME) in the hadronic phase [12] (see also Ref. [20]). Indeed, if one identifies $\partial_{t} \delta \pi^{0} / f_{\pi}$ with the "chirality imbalance" of chiral fermions defined by $\mu_{\mathrm{R}}-\mu_{\mathrm{L}} \equiv 2 \mu_{5}$, it exactly takes the form of the CME given, e.g., in Ref. [21]. This is similar to the fact that $\partial_{t} \theta$ plays the role of the chirality imbalance in the axion electrodynamics [22]. Note that the anomalous Hall effect of the form $\left(C / f_{\pi}\right) \boldsymbol{\nabla} \delta \pi^{0} \times \delta \boldsymbol{E}[12]$ is higher order in fluctuations and is ignored in the right-hand side of Eq. (7).
Taking the time derivative of Eq. (7), and then using the Faraday's law $\partial_{t} \boldsymbol{B}=-\boldsymbol{\nabla} \times \boldsymbol{E}$ and Eq. (6), we obtain

$$
\left(\partial_{t}^{2}-\nabla^{2}\right) \delta \boldsymbol{E}=\frac{C}{f_{\pi}}\left[\boldsymbol{\nabla}\left(\boldsymbol{\nabla} \delta \pi^{0} \cdot \boldsymbol{B}_{\mathrm{ex}}\right)-\partial_{t}^{2} \delta \pi^{0} \boldsymbol{B}_{\mathrm{ex}}\right] .
$$

It is clear from Eqs. (5) and (8) that, when $\delta \boldsymbol{E}$ and $\boldsymbol{\nabla} \delta \pi^{0}$ are perpendicular to $\boldsymbol{B}_{\mathrm{ex}}$, the photon does not receive any anomalous correction expressed by the right-hand sides, and the dispersion relation is just given by the usual one $\omega=k$. On the other hand, when $\delta \boldsymbol{E} \cdot \boldsymbol{B}_{\text {ex }} \neq 0$ and/or $\boldsymbol{\nabla} \delta \pi^{0} \cdot \boldsymbol{B}_{\mathrm{ex}} \neq 0$, the behavior of the photon is qualitatively modified by the anomalous effects.

\section{B. Dispersion relations}

Without loss of generality, we take the direction of the external magnetic field in the $z$ direction and set $\boldsymbol{B}_{\mathrm{ex}}=B_{\mathrm{ex}} \hat{z}$. As the simplest case, we assume that $\pi^{0}$ and photon are propagating in the $x$ direction; we also assume that the linear polarization $\delta \boldsymbol{E}$ is along the $z$ direction, $\delta \boldsymbol{E}=\delta E_{z} \hat{z}$. (From the argument above, the other linear polarization in the $y$ direction does not receive any correction.) The extension to more generic directions is straightforward. We look for the solutions of the form $\delta \pi^{0} \propto \mathrm{e}^{-\mathrm{i} \omega t+\mathrm{i} k x}$ and $\delta E_{z} \propto \mathrm{e}^{-\mathrm{i} \omega t+\mathrm{i} k x}$. From Eqs. (5) and (8), the equations of motion for $\delta \pi^{0}$ and $\delta E_{z}$ in momentum space can be summarized in the matrix equation $D_{i j} a^{j}=0$, where

$$
D \equiv\left(\begin{array}{cc}
\omega^{2}-k^{2} & \alpha \\
\alpha \omega^{2} & \omega^{2}-k^{2}
\end{array}\right), \quad \boldsymbol{a} \equiv\left(\begin{array}{l}
\delta \pi^{0} \\
\delta E_{z}
\end{array}\right) .
$$

Here we defined

$$
\alpha \equiv \frac{C B_{\mathrm{ex}}}{f_{\pi}}
$$

We can derive the particle spectrum and dispersion relations by the diagonalization of $D$. As a result, we obtain one gapless mode with the quadratic dispersion relation and one gapped mode,

$$
\omega=\frac{k^{2}}{\alpha}+O\left(k^{4}\right), \quad \omega=\alpha+O\left(k^{2}\right),
$$

respectively.

Without the external magnetic field, we originally have three gapless modes-photon with two polarizations and $\pi^{0}$. This result shows that the mixing between the photon with one of the polarizations and $\pi^{0}$ due to the WZW term leads to one gapless mode with the quadratic dispersion relation (while the photon with the other polarization remains unchanged and has the linear dispersion relation). This phenomenon looks seemingly similar to the emergence of the type B NG modes, whose previous examples 
include magnons in ferromagnets and $\mathrm{NG}$ modes in relativistic Bose-Einstein condensation [6,7].

\section{Temporal gauge}

It is easy to show that the nonrelativistic gapless mode in Eq. (11) can also be understood as a type-B NG mode in a particular choice of gauge- the temporal gauge $A_{t}=0$. In this gauge, the WZW term in Eq. (4) to the second order in fluctuations reduces to

$$
\mathcal{L}_{\text {mix }}=\alpha \pi^{0} \partial_{t} A_{z} .
$$

At sufficiently low energy, the kinetic terms for $\pi^{0}$ and $F^{\mu \nu}$ are negligible compared with this mixing term. In this regime, we can see from Eq. (12) that $A_{z}$ and $\pi^{0}$ are canonically conjugate degrees of freedom, and the corresponding NG mode must be just one, but not two, and hence, it is the type- $\mathrm{B}$ mode.

Note here that, without the background magnetic field, the leading term in the WZW term is third order in fluctuations of $\boldsymbol{E}, \boldsymbol{B}$ and $\pi^{0}$, and then $A_{z}$ and $\pi^{0}$ would not be canonically conjugate. This may simply be understood from the fact that a type-B NG mode could not appear in the absence of the explicit breaking of Lorentz invariance when the background magnetic field is turned off.

Note also that the structure of Eq. (12) is similar to the Lagrangians considered in Refs. $[6,7,23]$. However, the nonrelativistic gapless mode here is distinct from the previous examples of type-B NG modes in that this is an NG mode not associated with a usual symmetry for pointlike objects, but with a generalized global symmetry for extended objects.

\section{SYMMETRY ALGEBRA}

\section{A. Photon as a type-A NG mode of 1-form symmetries}

Before considering the relation of the nonrelativistic gapless mode in Eq. (11) to the SSB of a 1-form symmetry, we first recall, following Ref. [11], that the usual photon with the dispersion relation $\omega=k$ can be interpreted as a type-A NG mode associated with the SSB of 1-form symmetries.

In the vacuum without $\pi^{0}$ and $\boldsymbol{B}_{\mathrm{ex}}$, we have

$$
\begin{aligned}
& \partial_{\mu} F^{\mu \nu}=0, \\
& \partial_{\mu} \tilde{F}^{\mu \nu}=0,
\end{aligned}
$$

where Eq. (13) follows from the equation of motion in the vacuum and Eq. (14) follows from the Bianchi identity. These two equations can be regarded as the conservation laws for the 2-form currents, $j_{\mathrm{E}}^{\mu \nu} \equiv F^{\mu \nu}$ and $j_{\mathrm{M}}^{\mu \nu} \equiv \tilde{F}^{\mu \nu}$, which physically stand for the conservation laws of the electric and magnetic fluxes through a codimension-2 surface. They are the consequences of the 1-form symmetries, $A_{\mu} \rightarrow A_{\mu}+\lambda_{\mu}$ (electric symmetry) and $\tilde{A}_{\mu} \rightarrow$ $\tilde{A}_{\mu}+\tilde{\lambda}_{\mu}$ (magnetic symmetry) in Maxwell theory without matter, where $\tilde{F}_{\mu \nu}=\partial_{\mu} \tilde{A}_{\nu}-\partial_{n u} \tilde{A}_{\mu}$, and $\lambda$ and $\tilde{\lambda}$ are flat connections.

The operators charged under the 1-form electric and magnetic symmetries are the Wilson loop and 't Hooft loop,

$$
W(C) \equiv \exp \left(\mathrm{i} \int_{C} A\right), \quad T(C) \equiv \exp \left(\mathrm{i} \int_{C} \tilde{A}\right),
$$

respectively, where $C$ is a 1-dimensional closed loop. One can easily show that, in the vacuum (Coulomb phase), $\langle W(C)\rangle \neq 0$ and $\langle T(C)\rangle \neq 0$ under proper normalizations [11], and both the electric and magnetic symmetries are spontaneously broken. We then have

$\int_{\Sigma}\left\langle\left[j_{\mathrm{E}}^{0 i}(\boldsymbol{x}), W(C)\right]\right\rangle \neq 0, \quad \int_{\Sigma}\left\langle\left[j_{\mathrm{M}}^{0 i}(\boldsymbol{x}), T(C)\right]\right\rangle \neq 0$,

where the integral is taken over the codimension-2 surface $\Sigma$ normal to the $i$ direction. Equation (16) suggests that the total number of broken symmetry generators is six. However, not all of them are independent because of the commutation relation,

$$
\left[E^{i}(\boldsymbol{x}), B^{j}(\boldsymbol{y})\right]=-\mathrm{i} \epsilon^{i j k} \partial_{k} \delta(\boldsymbol{x}-\boldsymbol{y}),
$$

and Gauss's law $\boldsymbol{\nabla} \cdot \boldsymbol{E}=0$; as a result, only two of the broken symmetry generators are independent, which correspond to the two polarizations of photons. In this way, massless photons with the dispersion relation $\omega=k$ and with two polarizations can be understood as type-A NG modes associated with the SSB of the 1-form symmetries $[11,24,25]$.

\section{B. Type-B NG mode of the 1-form symmetry}

Let us turn to the original setup of QCD coupled to QED with $\boldsymbol{B}_{\text {ex }}$. In this case, not only the 1-form electric and magnetic symmetries, but also the $\mathrm{U}(1)_{\mathrm{A}}^{\tau_{3}}$ symmetry is spontaneously broken in the vacuum, and so we have the additional corresponding symmetry broken generator,

$$
Q_{5}=\int_{V} n_{5}(\boldsymbol{x}), \quad n_{5}=\bar{q} \gamma_{0} \gamma_{5} \tau^{3} q .
$$

Here $\int_{V}$ stands for the integral over the 3-dimensional spatial volume. However, $Q_{5}$ is not conserved in the presence of electromagnetic fields due to the triangle anomaly, and what is conserved instead is the combination,

$$
\tilde{Q}_{5} \equiv \int_{V}\left(n_{5}+C \boldsymbol{A} \cdot \boldsymbol{B}\right) .
$$

Here, the second term is the so-called magnetic helicity, which is gauge invariant under the proper boundary conditions. 
From the Lagrangian (4), the equation of motion (13) is modified to

$$
\partial_{\mu} F^{\mu \nu}=-\frac{C}{f_{\pi}} \tilde{F}_{\mathrm{ex}}^{\mu \nu} \partial_{\mu} \pi^{0},
$$

while the Bianchi identity (14) remains unchanged. Here we defined $\tilde{F}_{\mathrm{ex}}^{\mu \nu} \equiv\left(\delta^{\mu z} \delta^{\nu 0}-\delta^{\mu 0} \delta^{\nu z}\right) B_{\mathrm{ex}}$. Then, $j_{\mathrm{M}}^{\mu \nu}$ is again conserved, but $j_{\mathrm{E}}^{\mu \nu}$ itself is not. We can define the following conserved current by absorbing the right-hand side of Eq. (20) into the current:

$$
\tilde{j}_{\mathrm{E}}^{\mu \nu} \equiv F^{\mu \nu}+\frac{C}{f_{\pi}} \tilde{F}_{\mathrm{ex}}^{\mu \nu} \pi^{0} .
$$

We shall now work out the commutators between the symmetry broken generators, $\int_{\Sigma_{z}} \tilde{j}_{\mathrm{E}}^{0 z}, \int_{\Sigma_{z}} j_{\mathrm{M}}^{0 z}$, and $\tilde{Q}_{5}$. For this purpose, we need the commutation relations for the charges $\tilde{j}_{\mathrm{E}}^{0 i}, j_{\mathrm{M}}^{0 i}$, and $n_{5}$ :

$$
\begin{aligned}
{\left[\tilde{j}_{\mathrm{E}}^{0 i}(\boldsymbol{x}), j_{\mathrm{M}}^{0 j}(\boldsymbol{y})\right] } & =\mathrm{i} \epsilon^{i j k} \partial_{k} \delta(\boldsymbol{x}-\boldsymbol{y}), \\
{\left[\tilde{j}_{\mathrm{E}}^{0 i}(\boldsymbol{x}), n_{5}(\boldsymbol{y})\right] } & =-\mathrm{i} C\left(2 \tilde{F}^{0 i}-\tilde{F}_{\mathrm{ex}}^{0 i}\right) \delta(\boldsymbol{x}-\boldsymbol{y}),
\end{aligned}
$$

and the other commutators are vanishing. In order to derive Eq. (23), we used

$$
\left[\pi^{0}(\boldsymbol{x}), n_{5}(\boldsymbol{y})\right]=\mathrm{i} f_{\pi} \delta(\boldsymbol{x}-\boldsymbol{y}),
$$

and the anomalous commutator [26],

$$
\left[F^{0 i}(\boldsymbol{x}), n_{5}(\boldsymbol{y})\right]=-2 \mathrm{i} C \tilde{F}^{0 i} \delta(\boldsymbol{x}-\boldsymbol{y}),
$$

which is related to the triangle anomaly in relativistic quantum field theory. From Eq. (19) and the commutation relations (17), (23), and

$$
\left[A^{i}(\boldsymbol{x}), E^{j}(\boldsymbol{y})\right]=-\mathrm{i} \delta^{i j} \delta(\boldsymbol{x}-\boldsymbol{y}),
$$

we find

$$
\frac{1}{S\left(\Sigma_{z}\right)} \int_{\Sigma_{z}}\left\langle\left[\tilde{j}_{\mathrm{E}}^{0 z}(\boldsymbol{x}), \tilde{Q}_{5}\right]\right\rangle=-\mathrm{i} C B_{\mathrm{ex}},
$$

where the integral is taken over the codimension-2 surface $\Sigma_{z}$ normal to the $z$ direction and $S\left(\Sigma_{z}\right)$ is the area of $\Sigma_{z}$. Note that the right-hand side of Eq. (27) originates from the second term in Eq. (23) alone, since the anomalous contribution from the first term in Eq. (23) is absorbed as the magnetic helicity into $\tilde{Q}_{5}$. Equation (27) will play a key role in what follows.

We are now ready to apply the counting rule of the NG modes in Eqs. (1)-(3). Without the external magnetic field, we have three independent symmetry broken generators, $\int_{\Sigma_{z}} \tilde{j}_{\mathrm{E}}^{0 z}, \int_{\Sigma_{z}} j_{\mathrm{M}}^{0 z}$, and $\tilde{Q}_{5}$, which correspond to three type-A
NG modes. In the presence of the external magnetic field, Eq. (3) together with the anomalous commutator (27) dictates that $N_{\mathrm{B}}=1$, which corresponds to the NG mode with the quadratic dispersion in Eq. (11). Since $N_{\mathrm{BS}}=3$, Eq. (2) in turn leads to $N_{\mathrm{A}}=1$, which corresponds to the photon with the other polarization. This is consistent with the results above, demonstrating that the nonrelativistic NG mode in Eq. (11) is nothing but the type-B NG mode of the 1-form symmetry. ${ }^{1}$

We remark that, because the generator for the 1-form electric symmetry, $\int_{\Sigma_{\tilde{z}}} \tilde{j}_{\mathrm{E}}^{0 z}$, is restricted to the 2-dimensional surface $\Sigma_{z}$, the type-B NG mode appears only in the plane transverse to the external magnetic field. This is a feature specific to type-B NG modes of 1-form symmetries; in contrast, those of usual 0-form symmetries live in the whole 3-dimensional space.

\section{REALIZATION IN DIRAC SEMIMETALS}

So far, we have focused on QCD + QED in the external magnetic field as an example. As is clear from the derivation, our results above are universally applicable to the chiral symmetry broken phase of a massless Dirac fermion coupled to a U(1) gauge field in an external magnetic field.

One realization of our prediction in condensed matter systems is 3-dimensional Dirac semimetals, where charged massless Dirac fermions emergently appear as quasiparticles close to the band touching points. Since the effective coupling constant $\alpha_{\text {eff }} \equiv \alpha / v_{\mathrm{F}}$, with $\alpha \simeq 1 / 137$ being the coupling constant, can become strong due to the smallness of the Fermi velocity $v_{\mathrm{F}} \ll 1$ in candidate materials for Dirac semimetals, the strong Coulomb interaction may lead to the spontaneous breaking of $\mathrm{U}(1)$ axial symmetry and the corresponding NG mode-in a way somewhat similar to the QCD vacuum. In fact, a gapped phase (or insulating phase) for sufficiently large $\alpha_{\text {eff }}$ has been theoretically predicted using the strong-coupling expansion [27,28], Schwinger-Dyson equation [29], ladder approximation [30], and lattice Monte Carlo simulations [31,32]. When that happens, one can show from our argument above that the type-B NG mode of the 1-form symmetry with the quadratic dispersion relation emerges, when the external magnetic field is turned on. It would be interesting to search for such a new type of NG mode experimentally.

\footnotetext{
${ }^{1}$ This should be contrasted with the nonrelativistic photon that appears in the axion electrodynamics with a spatially varying and periodic $\theta$ term in the previous studies $[12,14,15]$. In those cases, the quadratic dispersion relation of photons follows from the commutator (17) in the presence of a nonvanishing $\langle\nabla \theta\rangle$ [13], which is different from our present situation. Also, the type-B NG mode appears in the 2-dimensional plane perpendicular to $\boldsymbol{B}_{\text {ex }}$ in our setup, while the nonrelativistic photon in Refs. [12,14,15] exists only in the 1-dimensional line in which $\theta$ is spatially varying.
} 


\section{CONCLUSION}

In this paper, we found a novel type-B NG mode of the 1form symmetry in a theory of strongly interacting massless Dirac fermion coupled to U(1) gauge field in an external magnetic field. We have shown that the existence of this mode is dictated by the anomalous commutator in the symmetry algebra. Although we limit ourselves to systems at zero temperature in this paper, one can show, similarly to Ref. [10] based on the Langevin-type low-energy effective theory, that this type-B NG mode appears even at finite temperature [33].

The presence of this type-B NG mode is expected to affect the low-energy dynamics dramatically. One example is the dynamic critical phenomenon, which is governed by the symmetries and low-energy degrees of freedom of a given system. In fact, one can show that the dynamic critical phenomenon at finite temperature affected by this NG mode is different from those known so far, providing a new dynamic universality class beyond the conventional classification. This will be reported elsewhere [33].

\section{ACKNOWLEDGMENTS}

We thank T. Brauner, Y. Hidaka, and R. Yokokura for useful discussions. We are especially grateful to Y. Hidaka for the explanation of the generalized global symmetries and for useful comments. N. S. is supported by JSPS KAKENHI Grant No. 17J04047. N. Y. is supported by JSPS KAKENHI Grant No. 16K17703 and MEXT-Supported Program for the Strategic Research Foundation at Private Universities, "Topological Science" (Grant No. S1511006).
[1] Y. Nambu, Phys. Rev. 117, 648 (1960).

[2] Y. Nambu and G. Jona-Lasinio, Phys. Rev. 122, 345 (1961).

[3] J. Goldstone, Nuovo Cimento 19, 154 (1961).

[4] J. Goldstone, A. Salam, and S. Weinberg, Phys. Rev. 127, 965 (1962).

[5] H. B. Nielsen and S. Chadha, Nucl. Phys. B105, 445 (1976).

[6] V. A. Miransky and I. A. Shovkovy, Phys. Rev. Lett. 88, 111601 (2002).

[7] T. Schäfer, D. T. Son, M. A. Stephanov, D. Toublan, and J. J. M. Verbaarschot, Phys. Lett. B 522, 67 (2001).

[8] H. Watanabe and T. Brauner, Phys. Rev. D 84, 125013 (2011).

[9] H. Watanabe and H. Murayama, Phys. Rev. Lett. 108, 251602 (2012).

[10] Y. Hidaka, Phys. Rev. Lett. 110, 091601 (2013).

[11] D. Gaiotto, A. Kapustin, N. Seiberg, and B. Willett, J. High Energy Phys. 02 (2015) 172.

[12] N. Yamamoto, Phys. Rev. D 93, 085036 (2016).

[13] Y. Hidaka (private communication).

[14] T. Brauner and S. Kadam, J. High Energy Phys. 03 (2017) 015.

[15] S. Ozaki and N. Yamamoto, J. High Energy Phys. 08 (2017) 098.

[16] Z. Qiu, G. Cao, and X. G. Huang, Phys. Rev. D 95, 036002 (2017).
[17] T. Brauner and S. V. Kadam, J. High Energy Phys. 11 (2017) 103.

[18] J. Wess and B. Zumino, Phys. Lett. 37B, 95 (1971).

[19] E. Witten, Nucl. Phys. B223, 422 (1983).

[20] K. Fukushima and K. Mameda, Phys. Rev. D 86, 071501 (2012).

[21] K. Fukushima, D. E. Kharzeev, and H. J. Warringa, Phys. Rev. D 78, 074033 (2008).

[22] D. E. Kharzeev, Ann. Phys. (Amsterdam) 325, 205 (2010).

[23] Y. Nambu, J. Stat. Phys. 115, 7 (2004).

[24] E. Lake, arXiv:1802.07747.

[25] D. M. Hofman and N. Iqbal, SciPost Phys. 6, 006 (2019).

[26] S. L. Adler and D. G. Boulware, Phys. Rev. 184, 1740 (1969).

[27] A. Sekine and K. Nomura, Phys. Rev. B 90, 075137 (2014).

[28] Y. Araki, Proc. Sci. LATTICE2015 (2016) 046.

[29] J. González, Phys. Rev. B 92, 125115 (2015).

[30] J. González, J. High Energy Phys. 10 (2015) 190.

[31] V. V. Braguta, M. I. Katsnelson, A. Y. Kotov, and A. A. Nikolaev, Phys. Rev. B 94, 205147 (2016).

[32] V. V. Braguta, M. I. Katsnelson, and A. Y. Kotov, Ann. Phys. (Amsterdam) 391, 278 (2018).

[33] N. Sogabe and N. Yamamoto (to be published). 